\title{
Heteronuclear Intermolecular Resonance-Assisted Hydrogen Bonds \\ The Structure of Pyrrole-2-Carboxyamide (PyCa)
}

\author{
Supplementary Material
}

\author{
Sławomir J. Grabowski, ${ }^{1,2}$ Alina Dubis, ${ }^{3}$ Marcin Palusiak ${ }^{1}$ and Jerzy Leszczynski ${ }^{2}$ \\ ${ }^{1}$ Department of Crystallography and Crystal Chemistry, University of Łódź, \\ ul. Pomorska 149/153, 90-236 Łódź, Poland \\ ${ }^{2}$ Computational Center for Molecular Structure and Interactions, Department of \\ Chemistry, Jackson State University, Jackson, MS 39217, USA \\ ${ }^{3}$ Institute of Chemistry, University of Biatystok, Al. J. Pitsudskiego 11/4, 15-443 \\ Biatystok, Poland
}

\begin{abstract}
The crystal and molecular structure of pyrrole-2-carboxamide (PyCa) determined by single crystal X-ray diffraction is presented. Molecular conformations of $\mathrm{PyCa}$ are also analyzed by FT-IR and NMR techniques. Additionally DFT calculations at the B3LYP/6$311++\mathrm{G}(\mathrm{d}, \mathrm{p})$ level of approximation are performed for dimers of $\mathrm{PyCa}$ and for related species. The existence of two tautomeric forms for the analyzed dimers differing in $\mathrm{H}$ bond motifs, N-H...O or O-H...N, is studied. The geometrical and energetic features of such H-bonds show that these interactions may be classified as intermolecular resonanceassisted hydrogen bonds (RAHBs). Additionally the Bader theory is applied to determine and to analyze bond critical points.
\end{abstract}

Key words: crystal and molecular structure, pyrrole-2-carboxamide, DFT calculations, hydrogen bond, the Bader theory, $\pi$-electron delocalization, FT-IR, NMR spectra 
Table. Bond distances $(\AA)$, bond angles and dihedral angles (degrees) for non-hydrogen atoms of PyCa molecules; estimated standard deviations are given in parentheses. All designations according to figure presented here (Fig 1.SUP).

\begin{tabular}{ll}
\hline O21-C21 & $1.2489(13)$ \\
C2-C3 & $1.3780(16)$ \\
N1-C2 & $1.3627(16)$ \\
C2-C21 & $1.4562(17)$ \\
N1-C5 & $1.3506(17)$ \\
C3-C4 & $1.3971(19)$ \\
N21-C21 & $1.3322(17)$ \\
C4-C5 & $1.3632(19)$ \\
N1-H1 & $0.891(15)$ \\
N21-H212 & $0.892(15)$ \\
N21-H211 & $0.832(15)$ \\
C2-N1-C5 & $109.56(10)$ \\
N1-C5-C4 & $108.44(11)$ \\
O21-C21-C2 & $121.73(10)$ \\
N21-C21-C2 & $116.65(10)$ \\
O21-C21-N21 & $121.57(11)$ \\
N1-C2-C21 & $121.42(9)$ \\
N1-C2-C3 & $107.21(10)$ \\
C3-C2-C21 & $131.29(10)$ \\
C2-C3-C4 & $107.51(10)$ \\
C3-C4-C5 & $107.28(10)$ \\
C5-N1-C2-C3 & $-0.54(13)$ \\
C5-N1-C2-C21 & $-177.54(10)$ \\
C2-N1-C5-C4 & $0.74(14)$ \\
N1-C2-C21-O21 & $9.74(16)$ \\
N1-C2-C21-N21 & $-172.50(10)$ \\
C3-C2-C21-O21 & $-166.45(11)$ \\
\hline
\end{tabular}




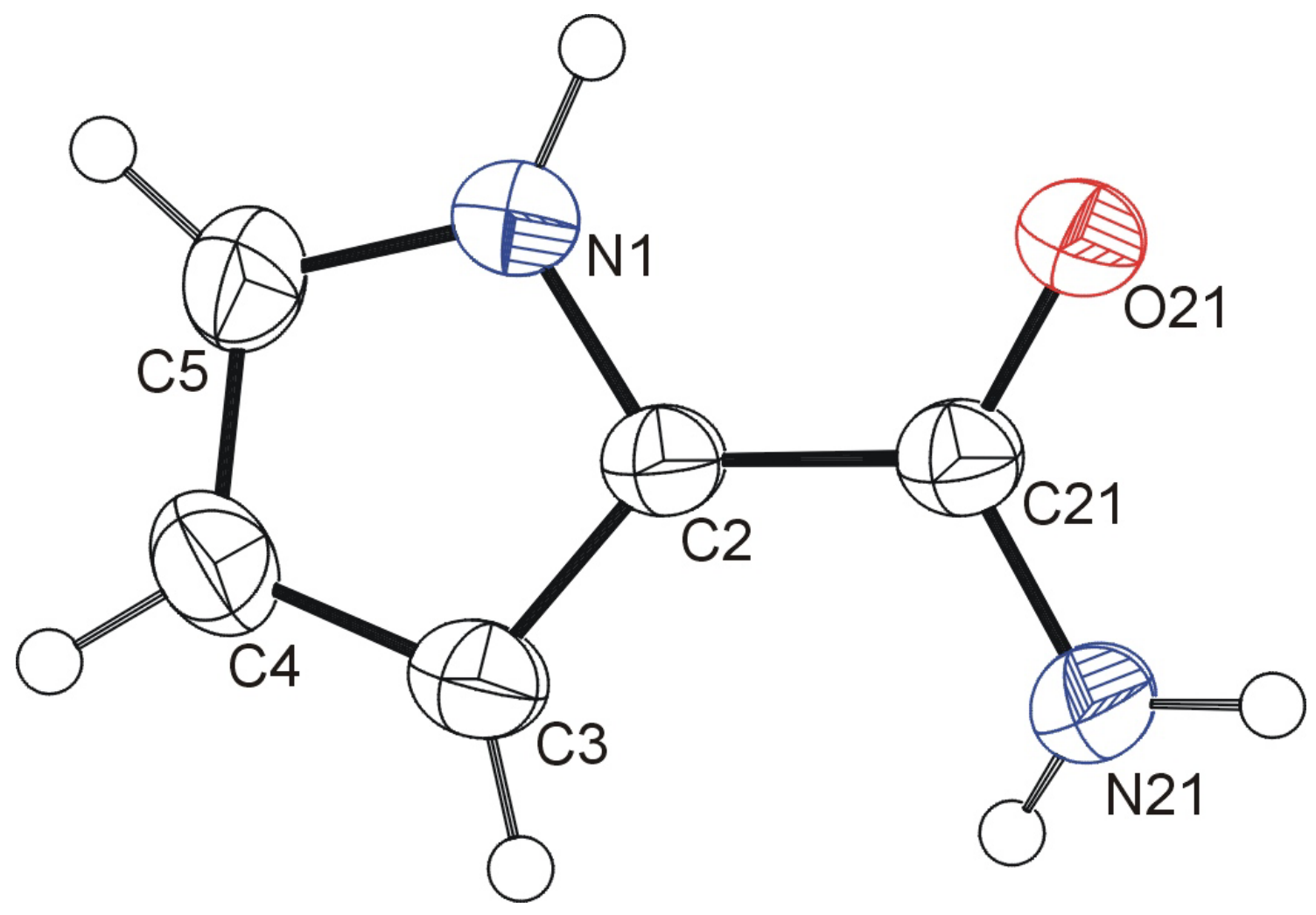

Figure 1.SUP Grabowski et al. 


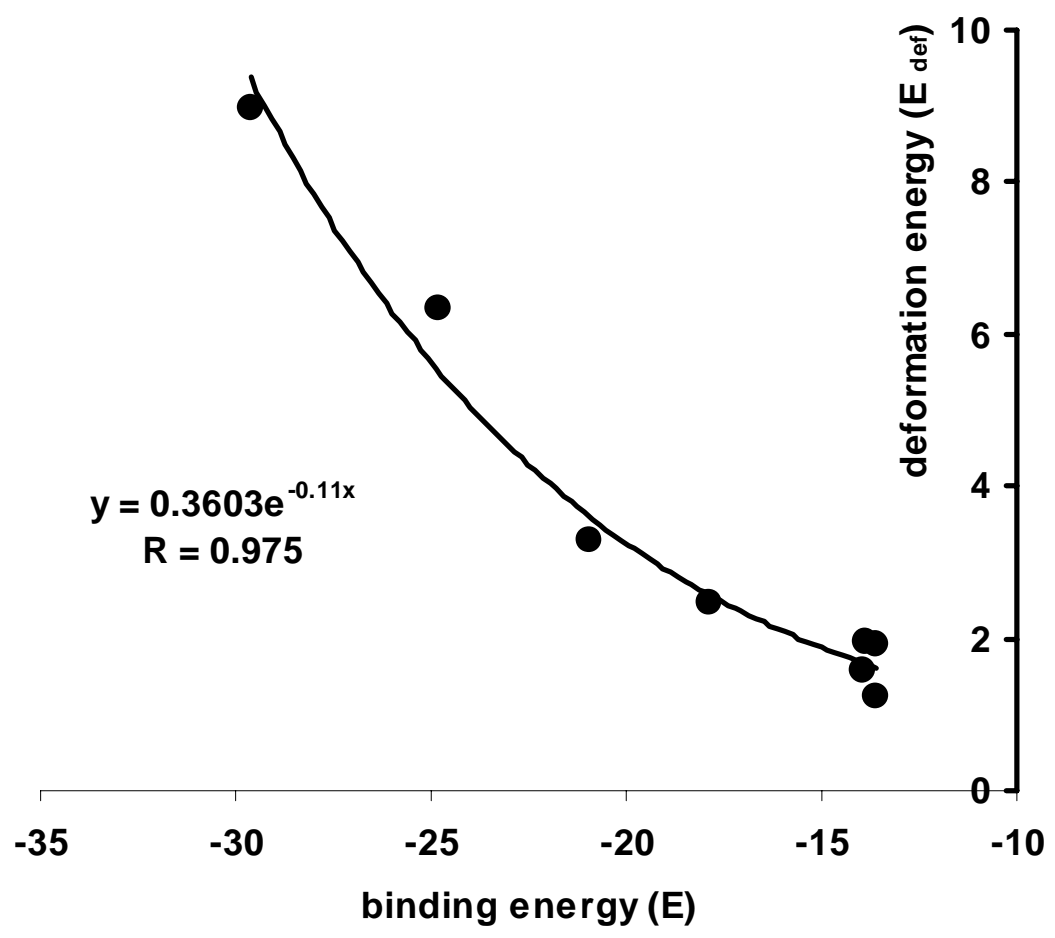

Figure 2.SUP Grabowski et al. 


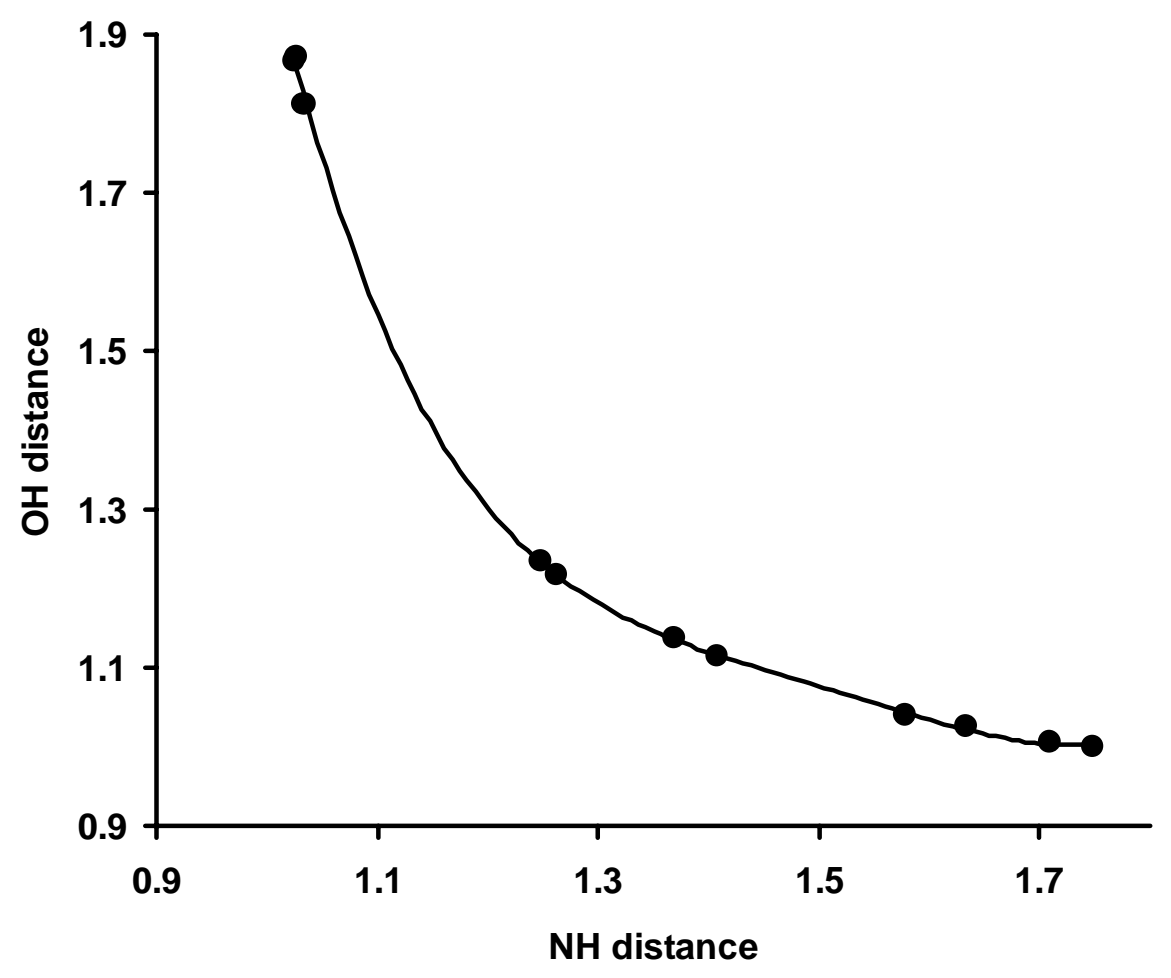

Figure 3.SUP Grabowski et al. 


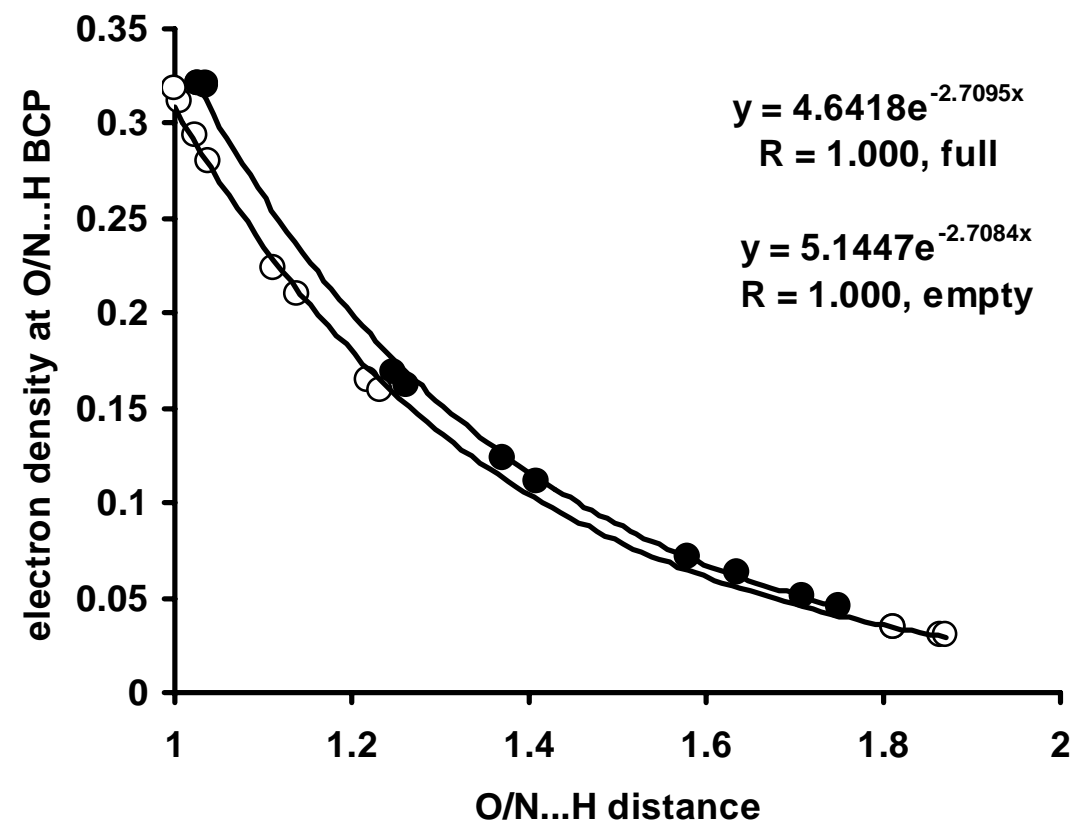

Figure 4.SUP Grabowski et al. 


\section{Figure captions}

Figure 1.SUP Molecular drawing of pyrrole-2-carboxyamide. Displacement ellipsoids are drawn at the $30 \%$ probability level.

Figure 2.SUP The binding energy calculated within the supermolecular approach versus the deformation energy; both energies are in $\mathrm{kcal} / \mathrm{mol}$.

Figure 3.SUP The relationship between $\mathrm{NH}(\mathrm{OH})$ bond length and $\mathrm{H} \ldots \mathrm{O}(\mathrm{H} \ldots \mathrm{N})$ intermolecular contact (all values in $\AA$ ).

Figure 4.SUP The dependencies between $\mathrm{NH}(\mathrm{H} \ldots \mathrm{N})$ and $\mathrm{OH}(\mathrm{H} \ldots \mathrm{O})$ (in $\AA$ ) on one side and the electron density at the corresponding BCP (in au) on the other side; empty circles correspond to $\mathrm{OH}$ bonds and $\mathrm{H} . . . \mathrm{O}$ distances while full squares designate $\mathrm{NH}$ and $\mathrm{H}$... $\mathrm{N}$ interactions. 